DOI: $10.1002 /(($ please add manuscript number $))$

Article type: (Full Paper)

\title{
In-Situ Scanning Electron Microscopy Observation of Growth Kinetics and Catalyst Splitting in Vapor-Liquid-Solid Growth of Nanowires
}

Xing Huang, Zhu-Jun Wang, Gisela Weinberg, Xiang-Min Meng, ${ }^{*}$ and Marc-Georg Willinger*

Dr. X. Huang, Z.-J. Wang, G. Weinberg, Dr. M.-G. Willinger

Department of Inorganic Chemistry, Fritz-Haber Institute of Max-Planck Society,

Faradayweg 4-6, 14195, Berlin, Germany.

E-mail: willinger@fhi-berlin.mpg.de

Dr. X. Huang, Prof. X.-M. Meng

Key Laboratory of Photochemical Conversion and Optoelectronic Materials, Technical Institute of Physics and Chemistry, Chinese Academy of Sciences, 100190, Beijing, P. R.

China

E-mail: mengxiangmin@mail.ipc.ac.cn

Keywords: in-situ ESEM, catalyst splitting, VLS, 1D semiconductor, cation substitution

In-situ observations during VLS growth of semiconductor nanowires in the chamber of an environmental scanning electron microscope (ESEM) are reported. For nanowire growth, a powder mixture of $\mathrm{CdS}$ and $\mathrm{ZnS}$ was used as source material and silver nanoparticles as metal catalyst. Through tracing growth kinetics of nanowires, we found that nanowires with a relatively bigger catalyst droplet on the tip grow faster. Intriguingly, we also found that the growth of nanowires can involve catalyst splitting: while the majority of catalyst remains at the nanowire tip and continues facilitating the growth, a portion of it is removed from the tip due to the splitting. It remains attached to the nanowire at the position where the splitting occurred and subsequently induces the growth of a nanowire branch. As far as we know, this is the first time that catalyst splitting is revealed experimentally in-situ. We propose that the instability of catalyst droplet caused by the volume increase is the main reason for the splitting. We believe that in-situ growth inside the ESEM could largely enrich our understanding on the metal-catalyzed VLS growth kinetics, which may open up more 


\section{WILEY-VCH}

opportunities for morphology controlled synthesis of 1D semiconductor nanowires in future study.

\section{Introduction}

One dimensional (1D) semiconductor nanowires have been widely studied for over two decades for their novel physical and chemical properties ${ }^{1-3}$ and a vast range of potential applications in electronics, ${ }^{4}$ piezoelectrics,${ }^{5}$ photovoltaics, ${ }^{6}$ mechanics, ${ }^{7}$ and sensors. ${ }^{8}$ With numerous research efforts devoted, tremendous progress has been made in the controlled synthesis of 1D semiconductor nanowires, including the control of diameter, ${ }^{9}$ length, ${ }^{10}$ composition, ${ }^{11}$ structure and orientation. ${ }^{12-16}$ Among all methodologies developed so far, the vapor-liquid-solid (VLS) process, first proposed by Wagner and co-workers for large whisker growth, ${ }^{17}$ has emerged as the most successful and widely adopted method for growing $1 \mathrm{D}$ semiconductor nanowires due to its great flexibility and high controllability. Three stages characterize the nanowire growth kinetics via a VLS mechanism, that is, alloying, nucleation and growth, respectively. ${ }^{18}$ As a characteristic of VLS mechanism, a metal catalyst, typically gold or silver, that mediates the mass transfer from the vapor phase to the growth front of a nanowire, is generally located at the tip of each growing nanowire. ${ }^{19}$ Recently, with the employment of in-situ techniques, particularly in-situ electron microscopy, ${ }^{18,20-25}$ significant breakthroughs have been achieved via real time observation of VLS growth kinetics, such as the revelation of heterogeneous nucleation, ${ }^{26}$ the observation of layer-by-layer growth mode, ${ }^{27}$ as well as the discovery of foreign metal catalyst loss channels. ${ }^{28}$ These findings are of fundamental interest in advancing our understanding of VLS mechanism and in turn are beneficial for the controlled fabrication of 1D semiconductor nanowires.

It is worth mentioning that most of the innovative findings, for example the results mentioned above, are based on investigations of simple systems. Typical systems are $\mathrm{Au} / \mathrm{Si}$ and $\mathrm{Au} / \mathrm{Ge}$, in which the $\mathrm{Au}$ serves as the metal catalyst while $\mathrm{Si}_{2} \mathrm{H}_{6}{ }^{26}$ and $\mathrm{Ge}_{2} \mathrm{H}_{6}{ }^{29}$ (or $\mathrm{SiH}_{4}$ and $\mathrm{GeH}_{4}$ ) serve as gas precursors for unitary nanowire growth. In other systems, however, things could 


\section{WILEY-VCH}

be much more complicated or even fundamentally different in some aspects. For instance, a recent study on self-catalyzed VLS growth of sapphire nanowires by in-situ TEM revealed that the layer-by-layer growth is accomplished by interfacial diffusion of oxygen from the triple junction, ${ }^{30}$ which is very different from the typical VLS mass transport mediated through the liquid phase. In order to expand our fundamental understanding of the VLS mechanism, it is therefore of importance to include other material systems, such as binary semiconductors, in the study of VLS growth kinetics.

Unlike the aforementioned unitary semiconductors whose precursors are supplied in gas phase and can therefore be fed into a TEM cell without causing serious contamination issues, the precursors of binary semiconductors, such as $\mathrm{ZnS}, \mathrm{CdS}$, and $\mathrm{PbSe}$, are in solid phase. Thus, they are not very suitable for in-situ TEM investigation. Although one may consider that the post growth and ex-situ characterizations can also help us understand the VLS growth mechanisms of such 1D semiconductors, they are in principle unable to provide a complete picture about the kinetics of VLS growth. Indeed, relevant details could be missed, leading to oversimplified or even misleading explanations of underlying growth mechanisms. Hence, a new approach that is capable of probing the growth kinetics of $1 \mathrm{D}$ binary semiconductors needs to be developed.

In this work, we demonstrate the in-situ observation on the growth kinetics of $\mathrm{ZnS}$ nanowires by employing an environmental scanning electron microscope (ESEM). The instrument is equipped with an FEI heating stage and a gas-feeding system. Thus the chamber of the ESEM can be used as an in-situ chemical vapor deposition (CVD) system that simultaneously enables electron microscopic investigation of the growth kinetics. Through studying the growth process of nanowires, we found that the growth speed of individual nanowires correlates with the size of the metal catalyst at the tip, which is in line with previous observations. ${ }^{31-33}$ More excitingly, we also demonstrate the first example of in-situ catalyst splitting during the process of nanowire growth. It occurs when the major part of the catalyst 


\section{WILEY-VCH}

remains at the tip, further catalysing the nanowire growth, while some portion of it is pulled off the tip and gets attached to the nanowire. Once it re-segregates as particles at the surface, the growth of nanowire branches is induced. Essentially, those kinetic events cannot be derived on the basis of post growth and ex-situ characterizations.

\section{Results and Discussion}

Figure 1a illustrates a schematic diagram of the experimental setup for nanowire growth, i.e., an ESEM equipped with a FEI heating stage. With the use of differential pumping systems, the instrument allows a gaseous environment in the specimen chamber while sustaining a high vacuum condition $\left(\sim 10^{-7} \mathrm{~Pa}\right)$ in the upper part of the column. An off-axis planar large field detector (LFD), positively biased at $80 \mathrm{~V}$, was employed for secondary electron (SE) collection during in-situ nanowire growth. In addition, a gas mixture of $\mathrm{H}_{2}$ : $\mathrm{Ar}(5 \%: 95 \%)$ that is normally used in a CVD process as the carrier gas was flowed into the chamber during the whole experimental process. The pressure inside the chamber can be set manually from $\sim 10$ $\mathrm{Pa}$ to $\sim 200 \mathrm{~Pa}$ in the low vacuum mode. The sample was prepared by loading a powder mixture of $\mathrm{CdS}$ and $\mathrm{ZnS}(\sim 2: 1)$ together with a small amount of silver glue into a crucible. The latter consists of Ag nanoparticles embedded in organic glue, which de-composes upon heating. It was used as a precursor for the Ag particles that catalyse nanowire growth. ${ }^{34,35}$ Figure $1 \mathrm{~b}$ shows a typical backscattered electron (BSE) image of the powder mixture placed in the crucible. In this imaging mode, the contrast is related to the atomic number $\mathrm{Z}$ of the specimen. The heavier CdS appears brighter than the $\mathrm{ZnS}$, which is also confirmed by EDX analysis (see Figure S1). Experiments were carried out by heating the sample up to 750$800^{\circ} \mathrm{C}$ in an $\mathrm{Ar}: \mathrm{H}_{2}$ atmosphere. Figure 2 shows a sequence of SEM images recorded from one nanowire during the in-situ growth (Schematic of nanowire growth is shown in Figure 2b). It is clear from the observation that the nanowire follows a typical VLS growth mode (Movie 1). The bright particles that are observed on the tip of each nanowire are the catalyst particles, which, according to the VLS growth mechanism, assist the nanowire growth by adsorption 


\section{WILEY-VCH}

and expulsion of material species. With time going, nanowires grew over 7 micrometers on the residual $\mathrm{ZnS}$ or $\mathrm{CdS}$ substrates (Figure S2). Interestingly, kinking events were also captured during nanowire growth (Movie 2), similar to kinked Si and Ge nanowires. As exemplified in Figure S3, nanowires can undergo multiple changes in growth direction. The high frequency of kinking occurrences and random distances between kinks indicate a high degree of kinetic forcing during nanowire growth. ${ }^{36}$ So far, mechanisms underlying the kinked growth are not well understood. Some effects, such as variations of temperature and partial pressure, ${ }^{37}$ hydrogen absorption, ${ }^{38}$ and catalyst jumping ${ }^{39}$ are known to be capable of inducing the kink formation. Apparently, far more experimental and theoretical investigations are required in order to arrive at a better understanding of the mechanisms behind kinking. In order to extract some information about the growth kinetics, the catalyzed growth of individual nanowires was tracked during the experiment (Movie 3). This is exemplified for three nanowires labelled as nanowire A (red), B (yellow) and C (green) in Figure 3. Since the three nanowires were growing in close proximity, it is assumed that they were exposed to very similar environmental conditions. The catalyzed growth of straight nanowires was followed for 2 minutes during which they reached a projected length (the length of nanowires on the projection plane, i.e., the image plane) of approximately $3.5 \mu \mathrm{m}$ at an average projected growth speed of $32.8 \mathrm{~nm} / \mathrm{s}, 29 \mathrm{~nm} / \mathrm{s}$ and $30.6 \mathrm{~nm} / \mathrm{s}$, respectively. It has been experimentally demonstrated in growth of Si nanowires that a larger catalyst can lead to a faster growth speed due to the Gibbs Thomson effect. ${ }^{31,32,40}$ Note that the initial size of the catalyst droplet pinned on the tip of nanowire $\mathrm{B}$ is smaller than those on the nanowires $\mathrm{A}$ and $\mathrm{C}$. Consequently, nanowire B should show a lower growth speed. However, in-situ observation also reveals that the catalyst B went through a significant diameter increase from $\sim 170 \mathrm{~nm}$ initially to $\sim 350 \mathrm{~nm}$ in the end (Figure 3b). This should also be taken into account when considering the influence of the catalyst diameter on the growth speed of nanowires. Therefore, to more fully elucidate the role of catalyst diameter in the nanowire growth, we artificially divide this kinetic process 


\section{WILEY-VCH}

into two stages, which are, stage I from 0 to $75 \mathrm{~s}$ and stage II from $75 \mathrm{~s}$ to $120 \mathrm{~s}$ respectively. The dividing point was chosen at $75 \mathrm{~s}$ because after that moment, the diameters of catalysts $\mathrm{A}$, $\mathrm{B}$, and $\mathrm{C}$ reached similar values. In this way, the projected growth speeds of nanowires $\mathrm{A}, \mathrm{B}$, and $\mathrm{C}$ are recalculated. They are $40.2 \mathrm{~nm} / \mathrm{s}, 31.7 \mathrm{~nm} / \mathrm{s}$ and $37.2 \mathrm{~nm} / \mathrm{s}$ in stage I and $20.4 \mathrm{~nm} / \mathrm{s}$, $24.4 \mathrm{~nm} / \mathrm{s}$ and $19.4 \mathrm{~nm} / \mathrm{s}$ in stage II, respectively. The overall decrease of the growth speeds from stage I to stage II can be attributed to the gradual exhaustion of source material. Through comparing the trend of projected growth speeds among three nanowires, it is interesting to find that nanowire B, the one used to be "slowest" in stage I, has become the "fastest" in stage II (Due to the projection, we can only compare the trend of growth speeds among different nanowires). Correlated with the size increase of catalyst B during nanowire growth, our result also show that the catalyst diameter has an important influence on the nanowire growth speed, that is, catalyst with relatively bigger size can lead to a faster growth speed. Figure $3 \mathrm{~b}$ plots the length of nanowires as well as diameter of catalysts as a function of time. One can see that those diagrams present a similar growth behavior, which may confirm our above consideration that nanowires grew under a similar environmental condition.

Post-growth characterization by transmission electron microscopy (TEM) and energydispersive X-ray spectrometry (EDX) revealed the structure and composition of the as-grown nanowires. Shown in Figure 4a is a high angle annular dark field scanning TEM (HAADFSTEM) image of an obtained nanowire, in which the different dark/bright contrast between the nanowire stem and the tip indicates a difference in the chemical composition. EDX elemental mapping of the nanowire (Figure $4 b-d$ ) unambiguously confirms that the tip is a silver nanoparticle while the stem is a $\mathrm{ZnS}$ nanowire. Figure 4e displays a high resolution TEM (HRTEM) image of the nanowire. Although stacking faults are exhibited, the lattice fringes, with $d$-spacings of 0.62 and $0.33 \mathrm{~nm}$, can be clearly resolved. They correspond to the (0001) and (01-10) planes of wurtzite ZnS, respectively and thus confirm the EDX data. Inset of Figure 4e shows a selected-area electron diffraction (SAED) pattern of the nanowire with 


\section{WILEY-VCH}

its [2-1-10] zone axis paralleling to the electron beam. Due to the presence of stacking faults, the diffraction spots along the [0001] direction are elongated. Nevertheless, the presence of one set of diffraction spots confirms the single crystalline nature of the nanowire. In addition, structural characterization of the metal tip was also performed, as shown in Figure S4. The HRTEM image and corresponding fast Fourier transformation (FFT) reveal that the tip nanoparticle has a cubic structure with the $[-130]$ zone axis paralleling to the electron beam. The lattice fringes with d-spacings of 0.12 and $0.2 \mathrm{~nm}$ fit well to (311) and (002) planes of silver. Now, one may wonder that with a co-evaporation of $\mathrm{ZnS}$ and $\mathrm{CdS}$ powder mixture, $\mathrm{Cd}$ should be detected as well in the resulting nanowires. Particularly, when considering the observation that the $\mathrm{CdS}$ particles vaporized much faster than the $\mathrm{ZnS}$, it might be possible that the initial grown nanowires are $\mathrm{CdS}$ nanowires or $\mathrm{Cd}$ rich $\mathrm{Cd}_{x} \mathrm{Zn}_{1-x} \mathrm{~S}$ nanowires. This anticipation can be strengthened by the fact that there were no nanowires produced if we only heat pure $\mathrm{ZnS}$ powder under otherwise similar conditions $\left(750-800{ }^{\circ} \mathrm{C}, \mathrm{H}_{2}: \mathrm{Ar}, 60 \mathrm{~Pa}\right)$. The slow evaporation rate of $\mathrm{ZnS}$ at the growth temperature is not capable of generating sufficient vapor species for the nucleation and subsequent growth. Hence, the above analysis leads to an assumption that there may exist another kinetic process accompanying with the nanowire growth, namely, cation substitution reaction of $\mathrm{Zn}^{2+}$ for $\mathrm{Cd}^{2+}$. We assume that the initial grown $\mathrm{CdS}$ (or $\mathrm{Cd}$ rich $\mathrm{Cd}_{x} \mathrm{Zn}_{1-x} \mathrm{~S}$ ) nanowires function as intermediate template for the formation of $\mathrm{ZnS}$ nanowires. Indeed, this is not the first case of cation substitution that occurred in the CdS-ZnS system. J. Park et al. has observed a complete conversion of ribbons of CdS to $\mathrm{ZnS}$ through a CVD process. ${ }^{41}$ Their study indicates that the mobility of cations plays an important role in determining the rate of the substitution reaction in the gas phase. With smaller radius and lighter mass, the mobility of $\mathrm{Zn}^{2+}$ is higher than that of $\mathrm{Cd}^{2+}$, which makes the cation exchange of $\mathrm{Zn}^{2+}$ for $\mathrm{Cd}^{2+}$ kinetically favourable. ${ }^{41} \mathrm{On}$ the other hand, considering the lower enthalpy for the formation of $\mathrm{ZnS}$ compared to that of $\mathrm{CdS}$, the chemical transition from $\mathrm{CdS}$ to $\mathrm{ZnS}$ is thermodynamically favourable as well. Giving those 


\section{WILEY-VCH}

reasons, it is rational to assume that the disappearance of $\mathrm{Cd}$ in nanowires is due to the cation exchange of $\mathrm{Zn}^{2+}$ for $\mathrm{Cd}^{2+}$. This process is illustrated schematically in Figure S5. In order to verify our above hypothesis by demonstrating the existence of intermediate $\mathrm{Cd}_{x} \mathrm{Zn}_{1-x} \mathrm{~S}$ nanowires, we carried out additional heating experiment with a short reaction time. Unfortunately, the intermediate $\mathrm{Cd}_{x} \mathrm{Zn}_{1-x} \mathrm{~S}$ phase was not clearly identified. Instead, TEM measurements showed that almost all the characterized nanowires consisted of $\mathrm{ZnS}$. This result, on the other hand, could indeed be an indication for a rapid exchange process of $\mathrm{Cd}^{2+}$ by $\mathrm{Zn}^{2+}$ during the nanowire growth. However, in a few cases it might be possible to say that a trace amount of $\mathrm{Cd}$ exists in the nanowires. Figure $4 \mathrm{f}$ and $\mathrm{g}$ shows a $\mathrm{ZnS}$ nanowire with a growth direction along the [2-1-10]. A portion of EDX spectrum recorded from the nanowire was displayed in Figure 4h (The full EDX spectrum is shown in Figure S6a). The peak centered at $2.98-3 \mathrm{keV}$ can be indexed to the Ag which probably wetted on the surface of nanowire, and the relatively weak peak at about 3.12-3.14 keV might be assigned to the $\mathrm{Cd}$. Since the amount of $\mathrm{Cd}$ is so low in nanowires that it is probably beyond the detective limitation of EDX in most cases.

It is well known that the morphology and structure of nanomaterials are strongly depending on the grown conditions. ${ }^{42-44}$ Some variations in local environment, such as temperature, pressure, and vapor concentration, could cause significant changes in growth kinetics, which in turn influence the morphology or structure of final nanostructures. ${ }^{45-48}$ In other ESEM experiments, probably due to the usage of higher amount of the silver catalyst in the crucible, growth kinetics of nanowires showed different behaviors. Instead of growth of single-stem nanowire as mentioned above, Figure 5 shows sequential SEM images of the growth of a branched nanowire (SEM images recorded during the $81 \mathrm{~s}$ are shown in Figure S7). Intriguingly, a direct splitting of catalyst droplet was observed during the growth. With the major part of catalyst advancing upwards, the expelled droplets stayed at the nanowire surface. During surface diffusion and precipitation the catalyst fragments remain at their original 


\section{WILEY-VCH}

position. Once they have condensed at the surface, the growth of nanowire branches is initiated (Movie 4). The schematic diagram of the whole process is shown in Figure 5b. To the best of our knowledge, this is the first example of direct in-situ observation of catalyst droplet splitting in a VLS process. In order to understand the kinetics of nanowire growth in more detail, we denote the growth process before splitting as stage I and the process afterwards as stage II, respectively. It can be calculated that the $\mathrm{ZnS}$ nanowire grew straight from 2.38 to $6.76 \mu \mathrm{m}$ within $86 \mathrm{~s}$ (Figure 5c), with an average projected growth speed of 55.9 $\mathrm{nm} / \mathrm{s}$ in stage I. This growth speed is much faster compared to those of the nanowires $\mathrm{A}, \mathrm{B}$, and $\mathrm{C}$ discussed above (Figure 3). This is most likely due to a higher concentration of vapor species in this experiment. In stage II, the volume of the catalyst droplet is reduced due to catalyst splitting (Figure 5c). Accordingly, the growth speed decreases from $55.9 \mathrm{~nm} / \mathrm{s}$ in stage I to $47.4 \mathrm{~nm} / \mathrm{s}$ in stage II. One may consider that the decrease of the growth speed could also be due to the decrease of vapor concentration in the local environment with growth time. However, the time dependent length of nanowire plotted in Figure 5c shows an approximate linear increase in stage I, which implies that the vapor concentration of growth species is indeed stable up to the splitting event. Figure $5 \mathrm{~d}$ shows a plot of growth speed of nanowire as a function of time derived from the Figure $5 \mathrm{c}$. It is clear that the speed of nanowire growth decreased down sharply after occurrences of catalyst splitting.

We now address the possible reasons for the catalyst splitting in the VLS process. The first one would be that surface defects may generate on the nanowire surface during growth. They could act as so called "trapping sites" and retain parts of the catalyst and induce the splitting. ${ }^{49}$ We have observed that after the splitting, the catalyst that remained on the surface of nanowire would first diffuse away within a few seconds and then condensed again right on the

original position from which the diffusion occurred (Figure 5a and Movie 4). The observation of confinement of catalyst to the position, to some extent, indicates the existence of "trapping sites" on the surface of nanowire. 


\section{WILEY-VCH}

The second reason for the catalyst splitting resides in the instability of catalyst droplet ascribed from the volume increase during the kinetic process. As illustrated in the growth model shown in Figure 6, the catalyst droplet that sits on the nanowire tip is balanced by the forces of liquid-vapor surface tension $\left(\sigma_{\mathrm{lv}}\right)$, solid-vapor surface tension $\left(\sigma_{\mathrm{sv}}\right)$ and the liquidsolid interface tension $\left(\sigma_{1 \mathrm{~s}}\right)$, respectively. ${ }^{50}$ Under an equilibrium condition, surface tension values of $\sigma_{\mathrm{lv}}, \sigma_{\mathrm{sv}}$ and $\sigma_{\mathrm{ls}}$ follow a relation of $-\sigma_{\mathrm{lv}} \sin \beta+\sigma_{\mathrm{ls}}=\sigma_{\mathrm{sv}}$ in the vertical direction. This indicates that as long as the net surface force on the droplet satisfies the relation of $-\sigma_{l v} \sin \beta+$ $\sigma_{\mathrm{ls}} \geq \sigma_{\mathrm{sv}}$, the droplet will maintain at the tip of nanowire during growth (Figure 6a). However, due to the exceeding amount of silver species in the local environment, the catalyst droplet would grow through adsorption, which leads to an increase of contact angle $\beta$. As a consequence, the vertical net force components of the droplet, $-\sigma_{l v} \sin \beta$, would be decreased with the increase of angle $\beta$. When it reaches a value that $-\sigma_{\mathrm{lv}} \sin \beta+\sigma_{\mathrm{ls}}$ is less than $\sigma_{\mathrm{sv}}$, i.e., $\sigma_{l v} \sin \beta+\sigma_{\mathrm{ls}}<\sigma_{\mathrm{sv}}$, the net force on the droplet would be pointed down (Figure 6b). In order to re-establish the tension-force-balance in vertical direction, a portion of catalyst would be released from the original droplet (Figure 6c).

Interestingly, in another case, a continuous discharge of catalyst droplet was observed, as shown in Figure S8 and Movie 5. The discharged catalyst formed a continuous layer on the nanowire surface and soon condensed to spherical droplets which subsequently induced the VLS growth of nanowire branches. In the present case, one can clearly see that the size of catalyst increases significantly before the occurrence of catalyst splitting, which is consistent to the above discussion. It can be predicted that as long as sublimation of the silver species is sufficient, the continuous dose of silver species to catalyst droplets would result in repeated catalyst splitting. As shown in Figure S8a (yellow), the catalyst on the nanowire tip regained in size and started to split again after the first continuous splitting discussed above.

Our observation of catalyst splitting in a VLS growth may give insight about the growth mechanism of branched or hyperbranched nanowires. In the case of PbSe hyperbranched 


\section{WILEY-VCH}

nanowires for example, fabricated through co-evaporation of $\mathrm{PbSe}$ with a small amount of $\mathrm{In}_{2} \mathrm{Se}_{3}$ powder in $\mathrm{N}_{2} / \mathrm{H}_{2}(95 \% / 5 \%)$ gas mixture, Cui et al. proposed that indium vapor, from evaporation of $\mathrm{In}_{2} \mathrm{Se}_{3}$, condensed as nanoscale liquid droplets on substrate and induced the nucleation and growth of pristine PbSe nanowires. ${ }^{51}$ Later, indium vapor nucleated onto the preformed $\mathrm{PbSe}$ nanowires and prompted the growth of additional nanowire branches. Alternately, Jin et al. synthesized $\mathrm{PbSe}$ hyperbranched nanowires using $\mathrm{PbCl}_{2}$ and $\mathrm{S} / \mathrm{Se}$ as precursors under hydrogen flow. ${ }^{49}$ They considered that the in-situ generated $\mathrm{Pb}$ due to reduction of $\mathrm{PbCl}_{2}$ by hydrogen serves as VLS catalysts for the growth of primary nanowires, and the continually produced $\mathrm{Pb}$ catalysts would later deposit along the length of the existing nanowires and lead to the nanowire branches growth. In general, they both believe that it is the condensations of the catalysts on the preformed nanowires that lead to the nucleation and growth of nanowire branches. However, according to our observation, the catalyst splitting can occur in a metal-catalysed VLS growth process, and those catalysts derived from the splitting can also induce the growth of nanowire branches. Those findings exposed by in-situ approach, may enrich our understanding on the kinetic behavior of catalyst droplet in a VLS process, and opens up an alternative explanation for the growth of branched nanowires as well. The structure of a branched nanowire was examined by TEM. Figure 7a shows a low magnification TEM image of a branched nanowire. The dark nanospheres on the nanowire surface are metal catalysts, which, we assume, come from the condensation of silver vapor from environment during cooling. This observation may confirm our conjecture that the environment for the branched nanowire contained sufficient silver vapor during the growth process. Disappearance of the catalyst on the branch tip could be due to the preparation of TEM sample. The HRTEM investigation (Figure $7 \mathrm{~b}$ and $\mathrm{c}$ ) reveals that the branched nanowire is $\mathrm{WZ} \mathrm{ZnS}$ with its stem and branch growing along the [1-10-1] and [11-20] directions, respectively. Crystallographically, the angle between [1-10-1] and [11-20] is $90^{\circ}$ in WZ 


\section{WILEY-VCH}

structure, which is in line with the observation that the branch is perpendicularly to the stem of nanowire.

\section{Conclusion}

In summary, we investigated the kinetics of catalyst-assisted growth of $\mathrm{ZnS}$ nanowires by an in-situ ESEM approach for the first time. We found that the nanowire growth speed is related to the diameter of catalyst on the tip. Nanowires with relatively larger catalyst grow at faster rate. This observation is consistent with previous works. We also demonstrated that the catalyst splitting can be involved in a VLS process. It was observed that, while the major part of the catalyst remained at the tip and assisted the growth of nanowire continuously after splitting; the part that remained on the nanowire surface subsequently condenses as nanoscale spheres and induces the growth of nanowire branches. This observation gives important insight about the growth mechanism of branched nanowires or hyperbranched nanowires, which was perhaps, oversimplified previously. TEM characterization combined with EDX analysis indicated that the resulting nanowires are $\mathrm{ZnS}$ with a $\mathrm{WZ}$ structure. We believe that a substitution of $\mathrm{Zn}^{2+}$ for $\mathrm{Cd}^{2+}$ leads to the absence of $\mathrm{Cd}$ in the nanowires eventually, which accompanies with the nanowire growth spontaneously. In-situ growth inside the chamber of an ESEM provides direct insights about the growth kinetics, and may lead us to a better understanding of the growth mechanisms of 1D semiconductor nanowires. Although the current study has not yet reached in a controlled manner for the synthesis of branched nanowires or hyperbranched nanowires, hopefully it can shed some light on it and help us to attain this target in the future.

\section{Experimental Section}

In-situ growth

We carried out the in situ nanowire growth experiments inside the chamber of a commercial ESEM (FEI Quantum 200) equipped with a FEI heating stage and a gas supply unit. A powder mixture of $\mathrm{CdS}$ and $\mathrm{ZnS}(\sim 2: 1)$ as well as silver glue were loaded into a crucible, which was 


\section{WILEY-VCH}

mounted in the heating stage. Nanowire growth was performed at $750-800^{\circ} \mathrm{C}$ using a flow of Ar: $\mathrm{H}_{2}(95 \%: 5 \%)$ gas mixture at a chamber pressure of 70-100 Pa. The temperature was measured via a K-type thermocouple that was dipped into the powder. During the experiments, the ESEM was operated at an acceleration voltage of $12.5 \mathrm{kV}$. Images were recorded using the secondary electron signal collected by a large field detector (LFD) during nanowire growth.

\section{Measurement}

TEM images, HRTEM images and EDX mapping analysis of nanowire was recorded by an Philips CM200 FEG transmission electron microscope and an FEI aberration-corrected Titan 80-300 transmission electron microscope equipped with an energy-dispersive X-ray spectroscopy.

\section{Supporting Information}

Supporting Information is available from the Wiley Online Library or from the author.

\section{Acknowledgements}

We appreciate valuable discussions with Dr. Hui Wang and Dr. Guangwei She from Technical Institute of Physics and Chemistry, Chinese Academy of Sciences. We want to thank the financial support from the "Strategic Priority Research Program" of the Chinese Academy of Sciences (XDA09040203). X. Huang would also like to thank the financial support from "Doctoral Training Programme" of Max-Planck Society and Chinese Academy of Sciences.

Received: ((will be filled in by the editorial staff))

Revised: ((will be filled in by the editorial staff)) Published online: ((will be filled in by the editorial staff))

[1] Y. N. Xia, P. D. Yang, Y. G. Sun, Y. Y. Wu, B. Mayers, B. Gates, Y. D. Yin, F. Kim, Y. Q. Yan, Adv. Mater. 2003, 15, 353-389.

[2] N. P. Dasgupta, J. W. Sun, C. Liu, S. Brittman, S. C. Andrews, J. Lim, H. W. Gao, R. X. Yan, P. D. Yang, Adv. Mater. 2014, 26, 2137-2184.

[3] W. Lu, C. M. Lieber, J. Phys. D-Appl. Phys. 2006, 39, R387-R406.

[4] M. T. Bjork, H. Schmid, J. Knoch, H. Riel, W. Riess, Nat. Nanotechnol. 2009, 4, 103-107.

[5] Y. F. Hu, Y. L. Chang, P. Fei, R. L. Snyder, Z. L. Wang, ACS Nano 2010, 4, 1234-1240.

[6] E. C. Garnett, P. D. Yang, J. Am. Chem. Soc. 2008, 130, 9224-9225.

[7] E. Gil-Santos, D. Ramos, J. Martinez, M. Fernandez-Regulez, R. Garcia, A. San Paulo, M. Calleja, J. Tamayo, Nat. Nanotechnol. 2010, 5, 641-645.

[8] Y. Cui, Q. Q. Wei, H. K. Park, C. M. Lieber, Science 2001, 293, 1289-1292. 


\section{WILEY-VCH}

[9] M. S. Gudiksen, J. F. Wang, C. M. Lieiber, J. Phys. Chem. B 2001, 105, 4062-4064.

[10] B. Moshofsky, T. Mokari, Chem. Mat. 2013, 25, 1384-1391.

[11] J. Xiang, W. Lu, Y. J. Hu, Y. Wu, H. Yan, C. M. Lieber, Nature 2006, 441, 489-493.

[12] X. Huang, M. G. Willinger, H. Fan, Z. L. Xie, L. Wang, A. Klein-Hoffmann, F. Girgsdies, C. S. Lee, X. M. Meng, Nanoscale 2014, 6, 8787-8795.

[13] Y. Hu, K. Q. Peng, Z. Qiao, X. Huang, F. Q. Zhang, R. N. Sun, X. M. Meng, S. T. Lee, Nano Lett. 2014, 14, 4212-4219.

[14] H. Huang, X. M. Ren, X. Ye, J. W. Guo, Q. Wang, Y. S. Yang, S. W. Cai, Y. Q. Huang, Nano Lett. 2010, 10, 64-68.

[15] T. R. Kuykendall, M. V. P. Altoe, D. F. Ogletree, S. Aloni, Nano Lett. 2014, 14, $6767-$ 6773.

[16] H. Wang, J.-T. Wang, Z.-X. Cao, W.-J. Zhang, C.-S. Lee, S.-T. Lee, X.-H. Zhang, Nat. Commun. 2015, 6, 6412.

[17] R. S. Wagner, W. C. Ellis, Appl. Phys. Lett. 1964, 4, 89-90.

[18] Y. Y. Wu, P. D. Yang, J. Am. Chem. Soc. 2001, 123, 3165-3166.

[19] H. L. Wang, L. A. Zepeda-Ruiz, G. H. Gilmer, M. Upmanyu, Nat. Commun. 2013, 4, 10.

[20] N. Petkov, ISRN Nanotechnol. 2013, 893060-893060.

[21] S. Biswas, C. O'Regan, M. A. Morris, J. D. Holmes, Small 2015, 11, 103-111.

[22] M. Kolibal, T. Vystavel, P. Varga, T. Sikola, Nano Lett. 2014, 14, 1756-1761.

[23] S. Kodambaka, J. Tersoff, M. C. Reuter, F. M. Ross, Science 2007, 316, 729-732.

[24] E. A. Sutter, P. W. Sutter, ACS Nano 2010, 4, 4943-4947.

[25] Y. C. Chou, K. Hillerich, J. Tersoff, M. C. Reuter, K. A. Dick, F. M. Ross, Science 2014, 343, 281-284.

[26] B. J. Kim, J. Tersoff, S. Kodambaka, M. C. Reuter, E. A. Stach, F. M. Ross, Science 2008, 322, 1070-1073.

[27] C. Y. Wen, M. C. Reuter, J. Bruley, J. Tersoff, S. Kodambaka, E. A. Stach, F. M. Ross, Science 2009, 326, 1247-1250.

[28] J. B. Hannon, S. Kodambaka, F. M. Ross, R. M. Tromp, Nature 2006, 440, 69-71.

[29] A. D. Gamalski, P. W. Voorhees, C. Ducati, R. Sharma, S. Hofmann, Nano Lett. 2014, 14, 1288-1292.

[30] S. H. Oh, M. F. Chisholm, Y. Kauffmann, W. D. Kaplan, W. D. Luo, M. Ruhle, C. Scheu, Science 2010, 330, 489-493.

[31] M. T. Borgstrom, G. Immink, B. Ketelaars, R. Algra, E. Bakkers, Nat. Nanotechnol. 2007, 2, 541-544. 


\section{WILEY-VCH}

[32] J. Kikkawa, Y. Ohno, S. Takeda, Appl. Phys. Lett. 2005, 86, 123109.

[33] S. A. Dayeh, S. T. Picraux, Nano Lett. 2010, 10, 4032-4039.

[34] Y. Liang, Y. Tao, S. K. Hark, CrystEngComm 2011, 13, 5751-5754.

[35] J. Zhang, X. Qing, F. H. Jiang, Z. H. Dai, Chem. Phys. Lett. 2003, 371, 311-316.

[36] P. Madras, E. Dailey, J. Drucker, Nano Lett. 2009, 9, 3826-3830.

[37] F. Lenrick, M. Ek, K. Deppert, L. Samuelson, L. R. Wallenberg, Nano Res. 2014, 7, 1188-1194.

[38] N. Shin, M. F. Chi, M. A. Filler, ACS Nano 2014, 8, 3829-3835.

[39] K. W. Schwarz, J. Tersoff, S. Kodambaka, F. M. Ross, Phys. Rev. Lett., 2014, 113, 055501 .

[40] E. I. Givargizov, J. Cryst. Growth 1975, 31, 20-30.

[41] J. Y. Lee, D. S. Kim, J. H. Park, Chem. Mat. 2007, 19, 4663-4669.

[42] X. Huang, M. Wang, L. D. Shao, M. G. Willinger, C. S. Lee, X. M. Meng, J. Phys. Chem. Lett. 2013, 4, 740-744.

[43] B. Z. Tian, P. Xie, T. J. Kempa, D. C. Bell, C. M. Lieber, Nat. Nanotechnol. 2009, 4, 824829.

[44] P. X. Gao, Y. Ding, W. J. Mai, W. L. Hughes, C. S. Lao, Z. L. Wang, Science 2005, 309, 1700-1704.

[45] G. Z. Shen, Y. Bando, B. D. Liu, D. Golberg, C. J. Lee, Adv. Funct. Mater. 2006, 16, 410416.

[46] M. S. Kumar, D. Chhikara, K. M. K. Srivatsa, Cryst. Res. Technol. 2011, 46, 991-996.

[47] J. Y. Lao, J. Y. Huang, D. Z. Wang, Z. F. Ren, Nano Lett. 2003, 3, 235-238.

[48] W. J. An, E. Thimsen, P. Biswas, J. Phys. Chem. Lett. 2010, 1, 249-253.

[49] M. J. Bierman, Y. K. A. Lau, S. Jin, Nano Lett. 2007, 7, 2907-2912.

[50] V. Schmidt, J. V. Wittemann, S. Senz, U. Gosele, Adv. Mater. 2009, 21, 2681-2702.

[51] J. Zhu, H. L. Peng, C. K. Chan, K. Jarausch, X. F. Zhang, Y. Cui, Nano Lett. 2007, 7, 1095-1099. 


\section{WILEY-VCH}

\section{Figure Caption}

Figure 1. (a) Schematic illustration of the heating experiment carried out in the ESEM; (b) Top-view colorized BSE image of the powder mixture, showing CdS (magenta) and ZnS (cyan) particles.

Figure 2. (a) A sequence of SEM images exhibiting nucleation and growth of a nanowire; (b) Schematic illustration.

Figure 3. (a) In-situ SEM observation of kinetics of nanowires growth; (b) Plots of length of nanowires and diameter of catalysts as a function of time.

Figure 4. (a-d) HAADF-STEM image and corresponding EDX elemental mapping of a grown nanowire; (e) HRTEM image and the corresponding SAED pattern; (f) TEM image of a ZnS nanowire grown along [2-1-10] direction; (h) HRTEM image corresponding to (f); A portion (2.5-4 keV) of EDX spectrum from the $\mathrm{ZnS}$ nanowire, showing the presence of $\mathrm{Ag}$ and possible presence of Cd signal.

Figure 5. Kinetics of catalyst splitting: (a) In situ SEM observation of the catalyst splitting in the growth of branched nanowire; (b) Model of catalyst splitting and branch nanowire growth; (c) Length of nanowire and diameter of catalyst as a function of time; (d) Velocity of nanowire as a function of time.

Figure 6. Schematic diagram depicting the process of catalyst splitting.

Figure 7. (a) Low magnification TEM image of a branched nanowire; HRTEM images of (b) stem and (c) branch of the nanowire. 


\section{WILEY-VCH}

Figure 1
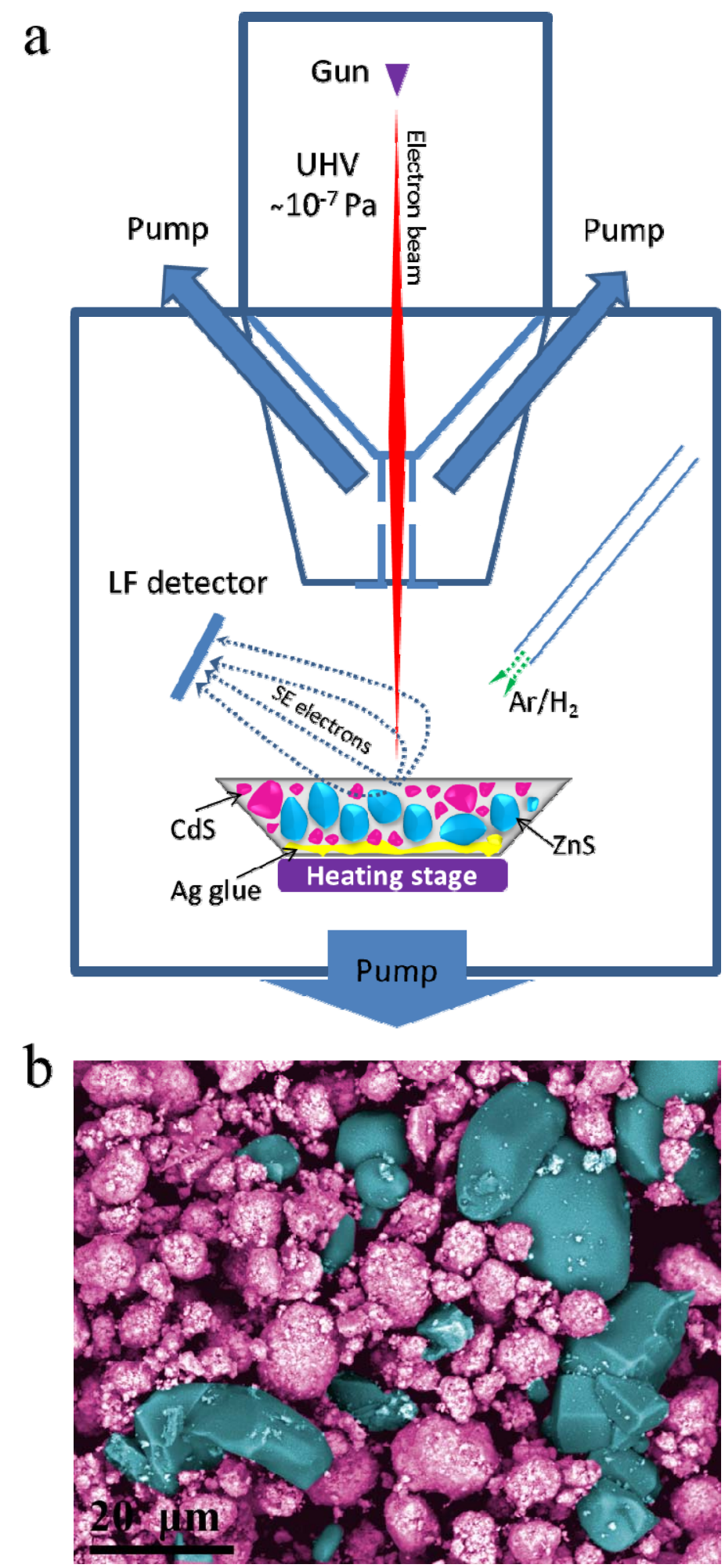


\section{WILEY-VCH}

Figure 2
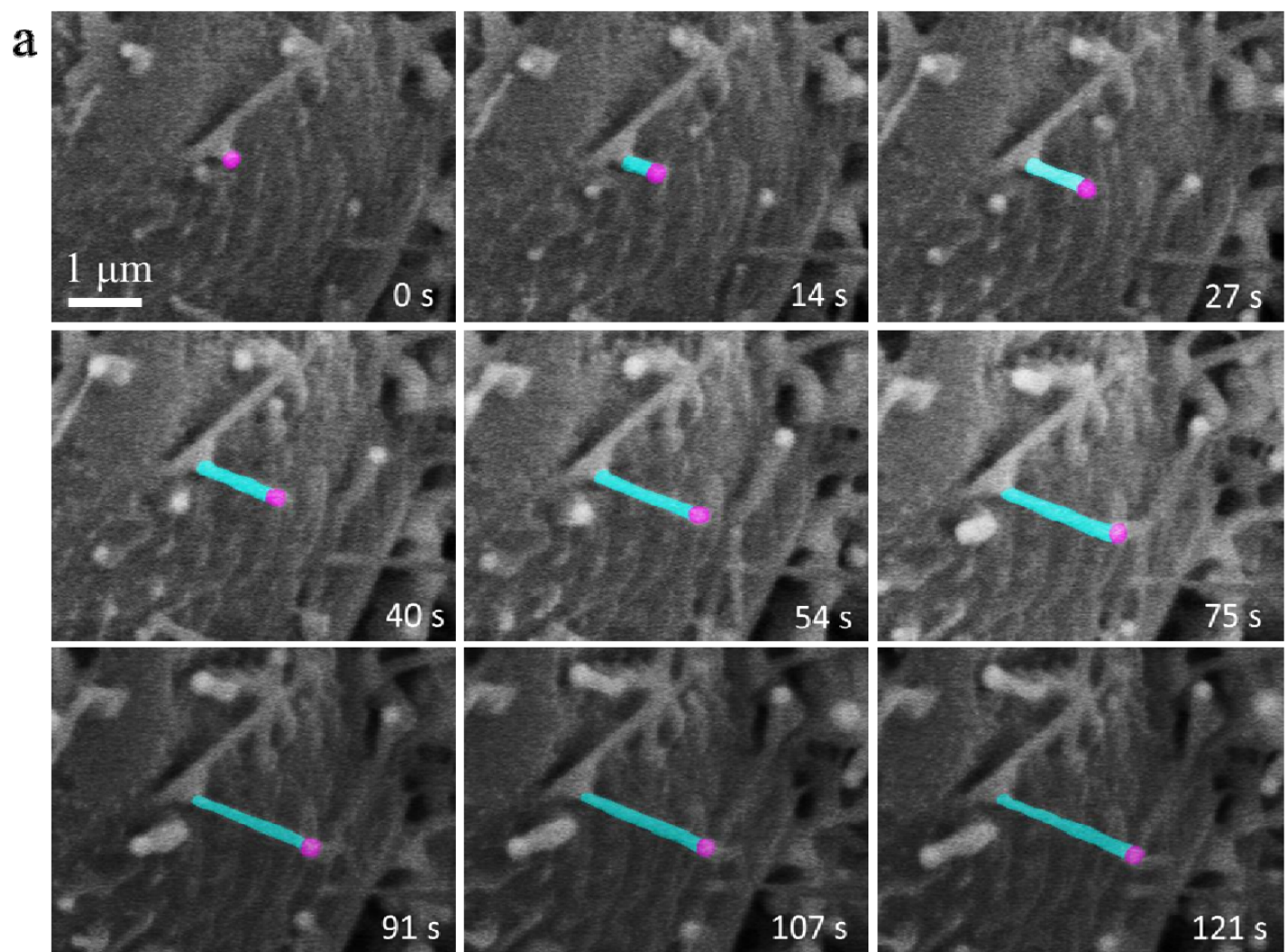

b

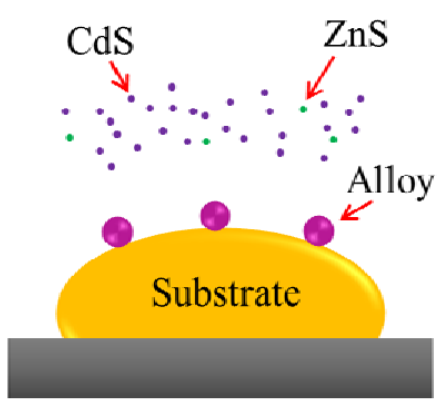

Alloying

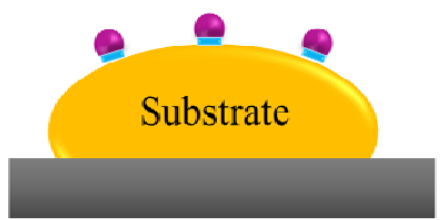

Nucleation

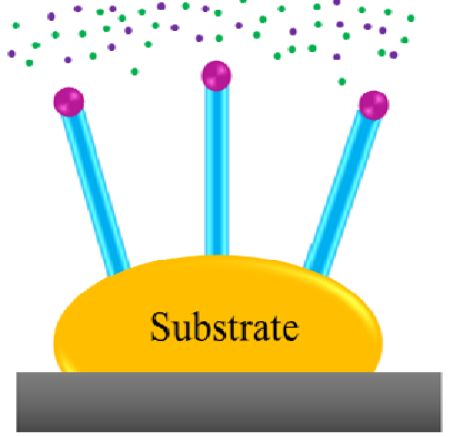

Growth 


\section{WILEY-VCH}

Figure 3

a
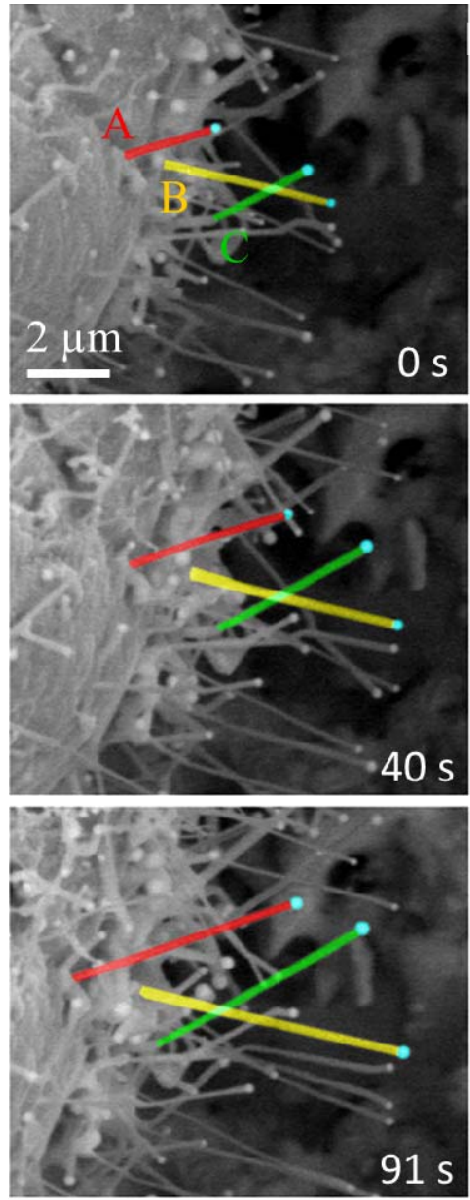

b

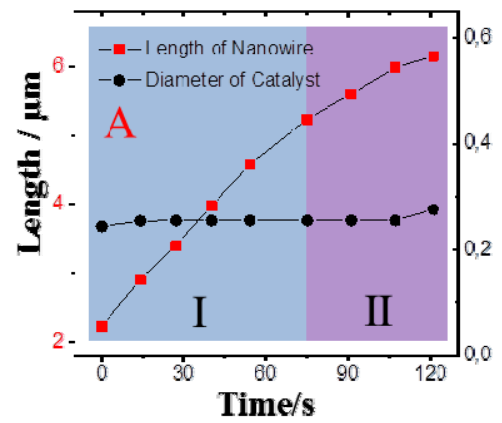

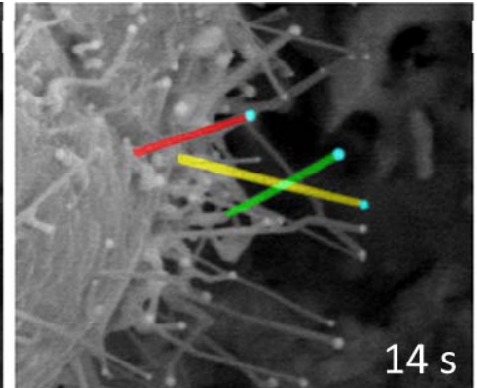
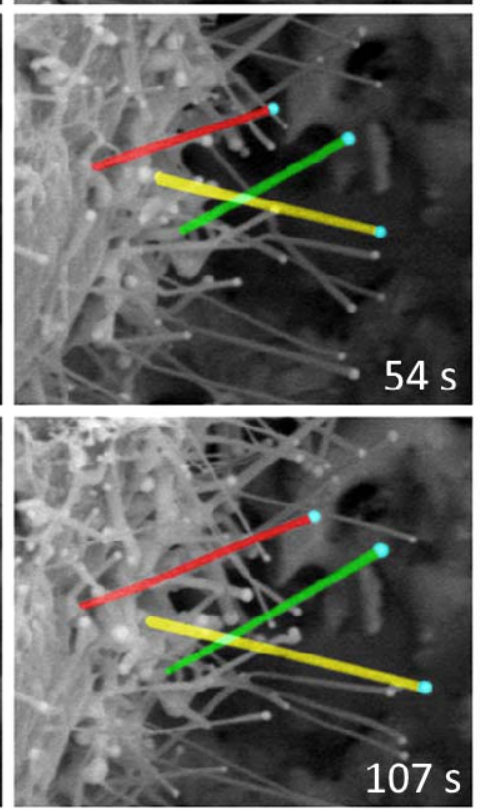

$107 \mathrm{~s} / \mathrm{f}, 121 \mathrm{~s}$

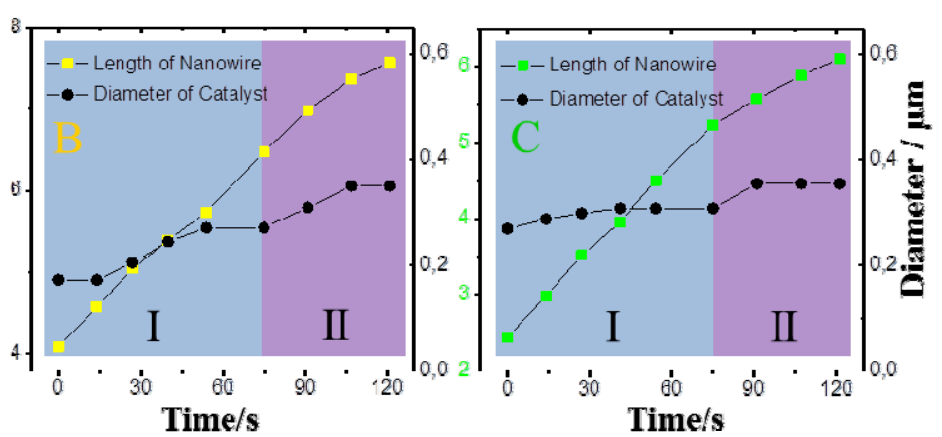




\section{WILEY-VCH}

Figure 4

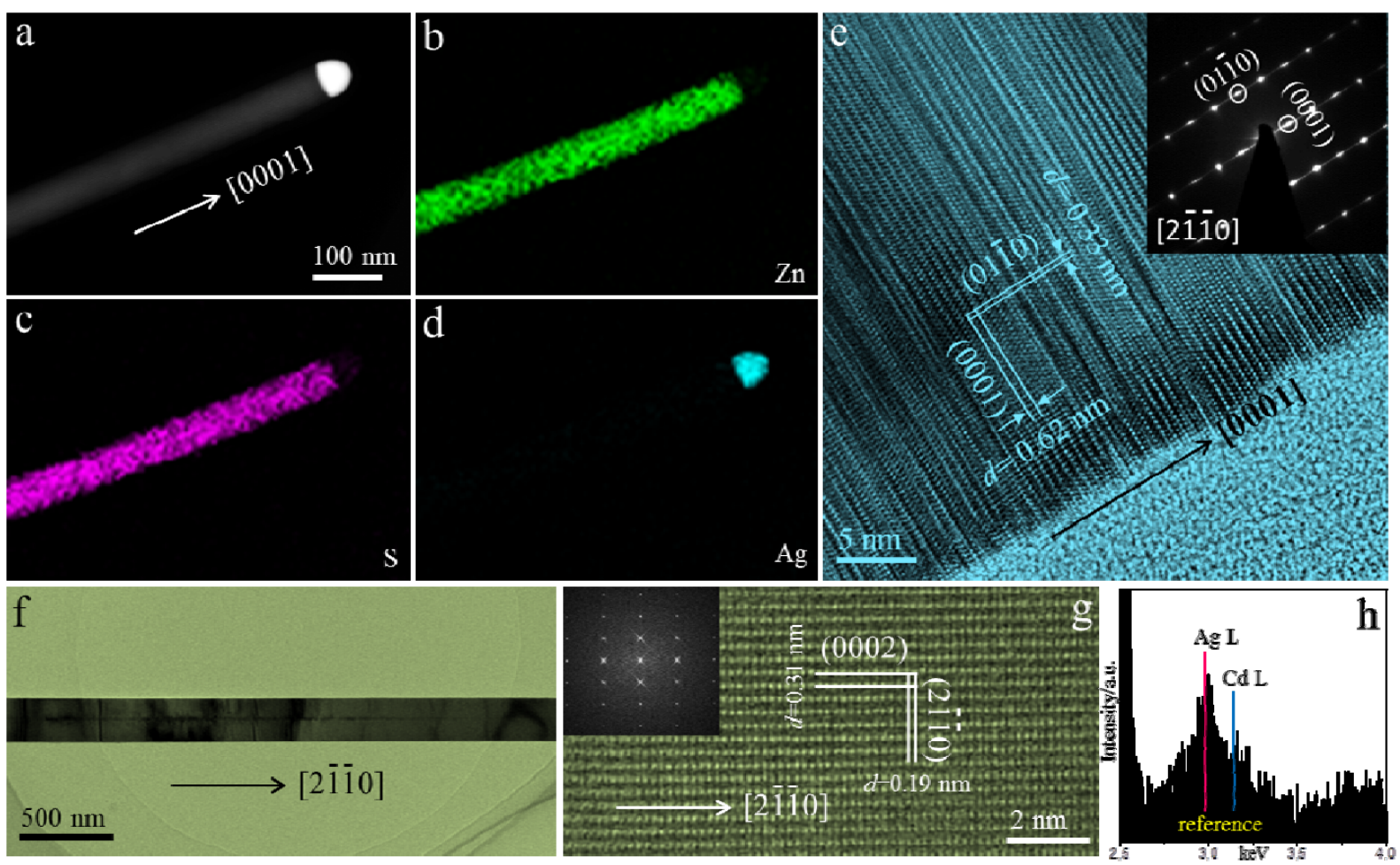




\section{WILEY-VCH}

Figure 5
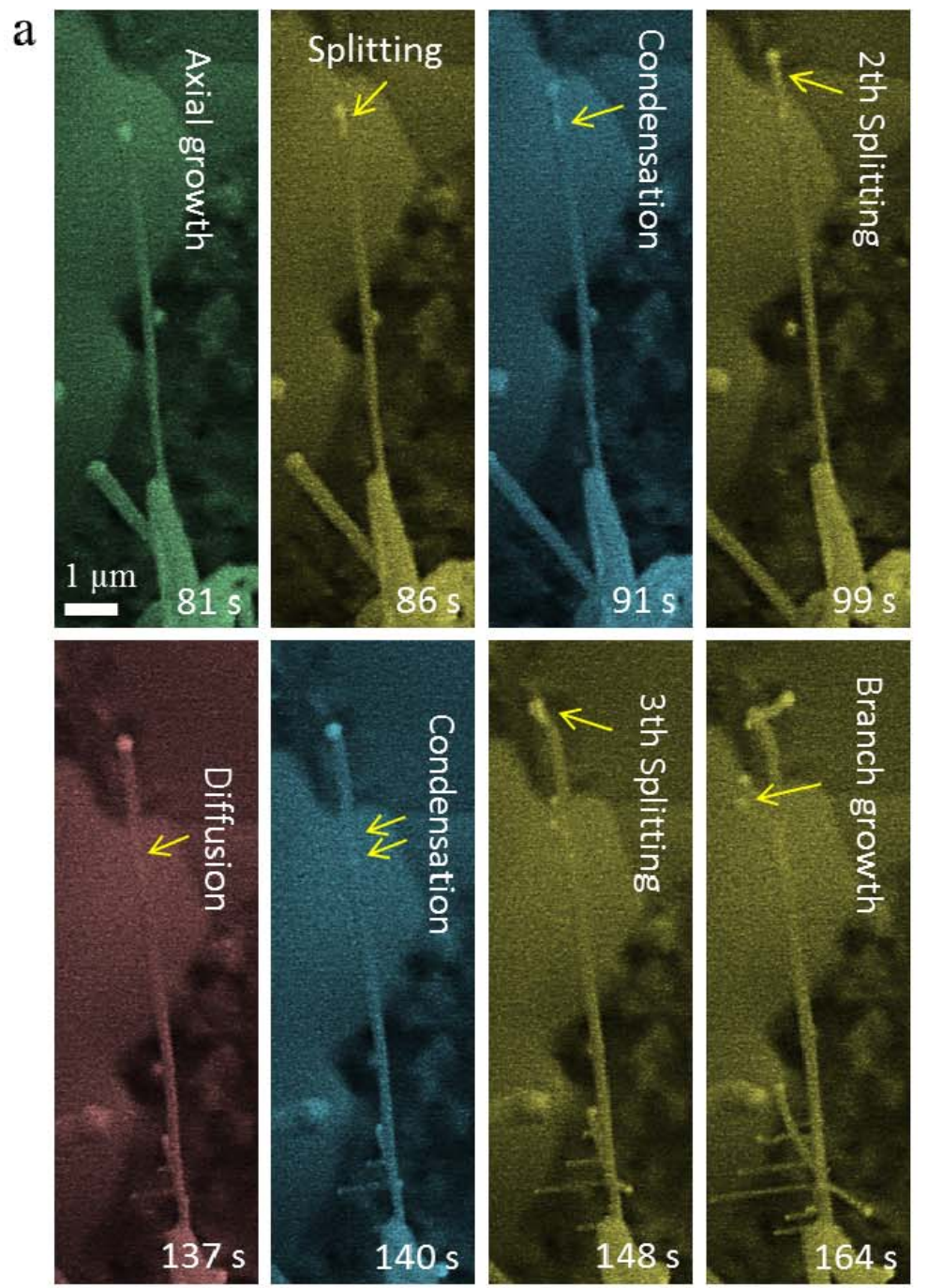

b
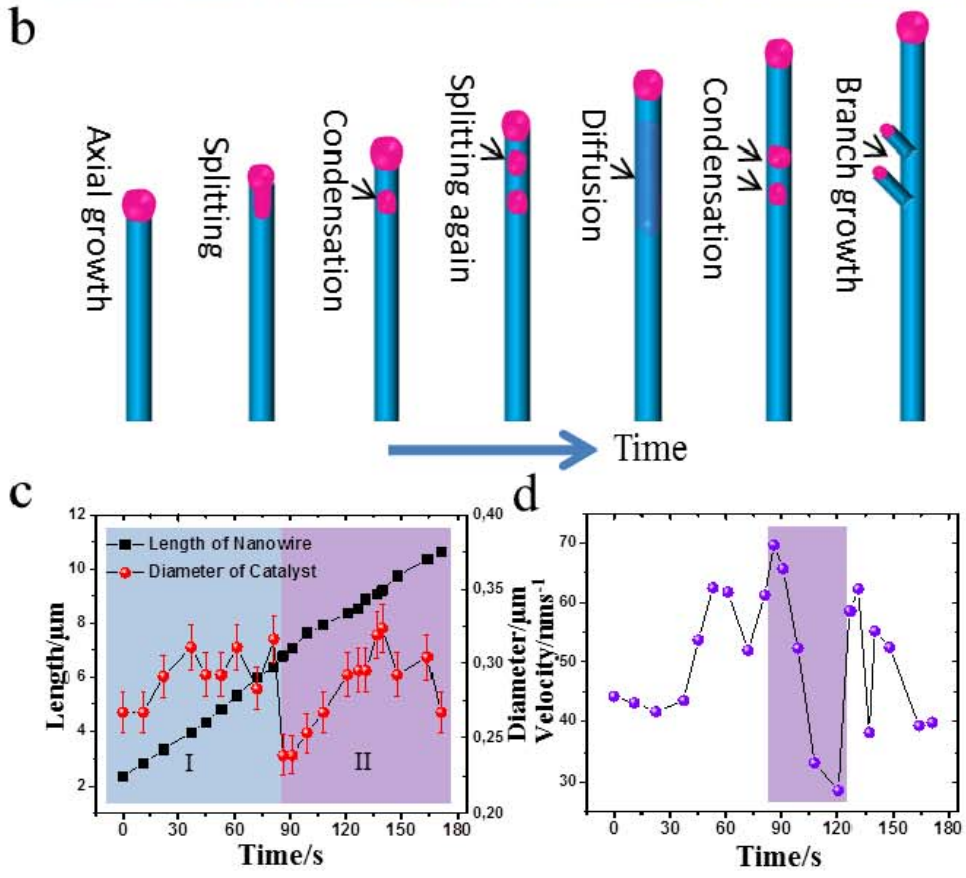


\section{WILEY-VCH}

Figure 6

a

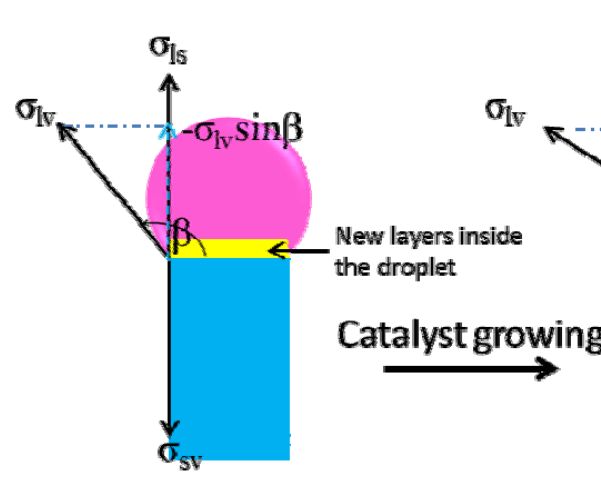

Stable b

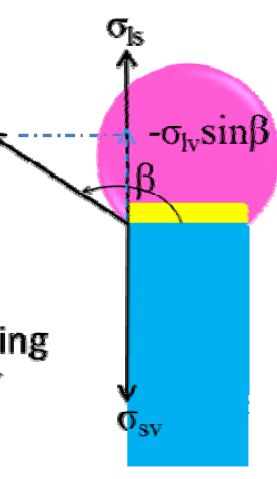

Unstable

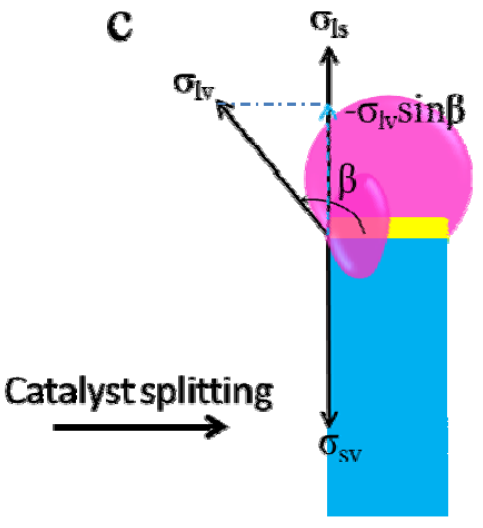

stable 


\section{WILEY-VCH}

Figure 7
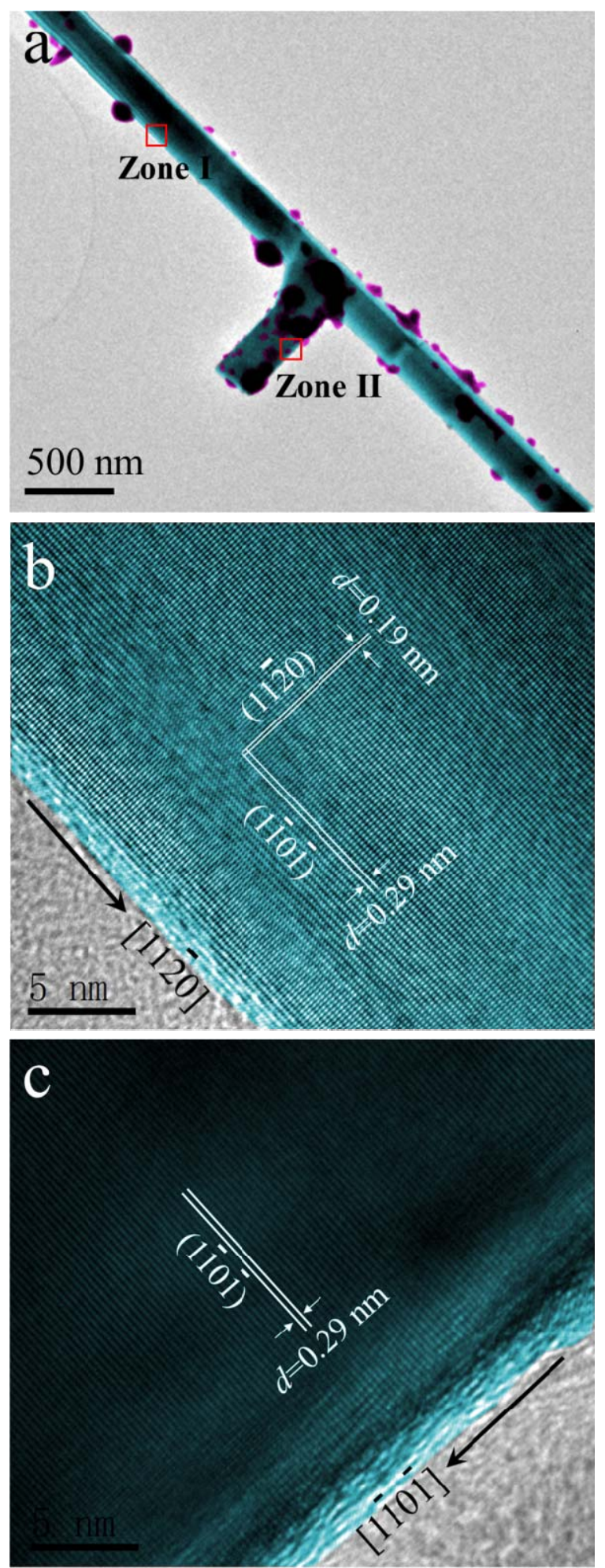


\section{WILEY-VCH}

\section{In-Situ Scanning Electron Microscopy Observation of Growth Kinetics and Catalyst Splitting in Vapor-Liquid-Solid Growth of Nanowires}

Xing Huang, Zhu-Jun Wang, Gisela Weinberg, Xiang-Min Meng, ${ }^{*}$ and Marc-Georg Willinger*

Keywords:

in-situ ESEM, catalyst splitting, VLS, 1D semiconductor, cation substitution

\section{ToC figure}

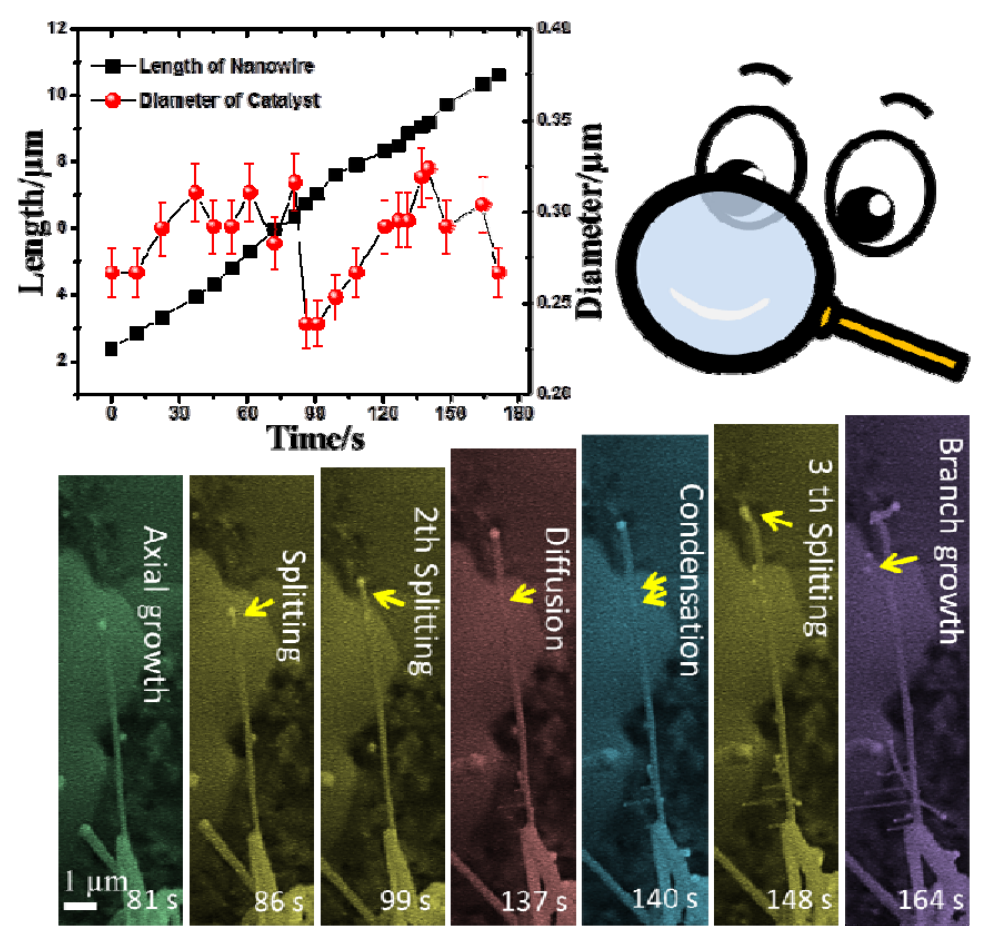

Catalyst splitting in vapor-liquid-solid growth kinetics is observed during in-situ growth of nanowires inside the chamber of a scanning electron microscope. The splitting occurs with the majority of catalyst remaining at the nanowire tip, further enabling nanowire growth, while the other portion remains attached to the nanowire and subsequently induces the growth of a nanowire branch. 